\title{
Commentary: Granins, Secretory Granule Biogenesis, and Transport
}

\author{
Y. Peng Loh • Angelo Corti
}

Published online: 22 May 2012

(C) Springer Science+Business Media, LLC (outside the USA) 2012

Secretory granules are the hallmark of secretory cells that secrete granins, peptide hormones, and enzymes for various physiological functions in endocrine, exocrine, and neurons. The granin family of proteins are highly acidic and are the major components in dense core secretory granules, including those in chromaffin cells. Granins include chromogranin (Cg) A, B, and secretogranin (Sg) II which are processed to various derived peptides. In this 16th International Symposium of Chromaffin Cell Biology, a number of papers focusing on the mechanisms of biogenesis and transport of secretory granules in chromaffin cells, other endocrine cells and astrocytes were presented. As well, new physiological roles of granin-derived peptides were discussed.

Maite Montero-Hadjadje (France) demonstrated that via its terminal domains, $\mathrm{CgA}$ induced granule biogenesis and generated a regulated secretory pathway in fibroblasts. These granules recruited cytoskeletal proteins, actin/myosin and microtubule motors to their surface and exhibited $\mathrm{Ca}^{++}$sensitive transport to the plasma membrane for release. Peng Loh (USA) showed that a C-terminal CgA-derived peptide, serpinin, upon being released after stimulation of the cells, upregulated the transcription of a protease inhibitor, protease nexin-1 (PN-1) in a cAMP-PKA-sp1 dependent pathway. This in turn stabilized and augmented the level of granule proteins in the Golgi complex, leading to enhanced granule biogenesis.

\section{Y. P. Loh $(\bowtie)$}

Section on Cellular Neurobiology, Program on Developmental Neuroscience, Eunice Kennedy Shriver National Institute of Child Health and Human Development, National Institutes of Health, Bethesda, MD 20892, USA

e-mail: lohp@mail.nih.gov

\section{A. Corti}

Tumor Biology and Vascular Targeting Unit, DIBIT-Division of Molecular Oncology, San Raffaele Scientific Institute, Milan 20132, Italy
Ricardo Borges (Spain) using chromaffin cells from $\mathrm{CgA}$ knockout (KO), $\mathrm{CgB} \mathrm{KO}$ and $\mathrm{Cg}$ double $\mathrm{KO}$ mice showed that the absence of granins led to changes in the accumulation and secretion of catecholamines. They concluded that these Cgs are highly efficient in facilitating monoamine and calcium accumulation and in the kinetics of exocytosis of dense core granules (for more details, see review by Borges group below). Seung Yoo (Korea) demonstrated the presence of inositol $1,4,5$, triphosphate (IP3) sensitive $\mathrm{Ca}^{++}$stores and three forms of IP3 receptors/channels in secretory granules in astrocytes, that presumably interacts with the $\mathrm{Cgs}$ to modulate $\mathrm{Ca}^{++}$ channels and $\mathrm{Ca}^{++}$release as in chromaffin granules.

Several papers were presented demonstrating the role of cytoskeletal network/proteins in granule transport to the plasma membrane for exocytosis. Luis Gutierrez (Spain) presented confocal microscopy and 3D reconstructed images revealing the complex F-actin structure at the cortex of chromaffin cells. He showed that this F-actin structure underwent reorganization during secretion to reposition the vesicles for exocytosis, (for more details, see their review below). Heidi de Wit (The Netherlands) recapped her extensive studies on the morphology of synaptic protein knockout cells at the electron microscopy level, suggesting that syntaxin, SNAP25 and synaptogamin form the minimal docking machinery. She also discussed the role of Munc 18 in reorganizing cortical F-actin for vesicle docking at the plasma membrane, prior to secretion, in chromaffin cells of embryonic mice. (See her review below). Frederic Meunier (Australia) described a novel phosphatidylinisitol $(4,5)$ biphosphate pathway that controls actin-mediated mobilization and translocation of secretory vesicles to the plasma membrane. Small GTPases such as the Rho GTPase family are involved in actin remodeling during exocytosis. Stephane Gasman (France) showed in chromaffin cells and PC12 cells that Rho A, which is associated with secretory 
granules, had a negative effect on exocytosis; whereas Rac1 and $\mathrm{Cdc} 42$ found in the subplasmalemmal area had a positive effect. Rho A seems to be involved in maintaining cortical actin network integrity by generating granule bound phosphoinositol 4-phosphate, Rac1 in stimulating phospholipase D-mediated phosphatidic acid formation and Cdc42 in activating actin filament formation at the exocytotic site; together they orchestrate exocytosis through controlling actin and lipid dynamics. Aniko Varadi (UK) demonstrated the essential role of another cytoskeletal protein, brain spliced isoform of Myosin Va (BR-MyoVa) in secretory granule transport to the plasma membrane. BR-MyoVa has a number of binding partners: granuphilin-a/b, Rab27a, Rab3A which are associated with secretory granules. She showed that disruption of granuphilin-a/b-BR-MyoVa interaction led to perinuclear accumulation of secretory granules and enhanced secretion of insulin in pancreatic beta cells. Nicolas Vitale (France) presented data showing that Tat, a protein released by HIV-infected cells, was actively internalized, and inhibited catecholamine secretion and large dense core granule exocytosis in chromaffin cells and PC12 cells, respectively. Tat inhibited neurosecretion through impairment of Annexin2-dependent plasma membrane recruitment of $\mathrm{Cdc} 42$, which prevented cortical actin reorganization and movement of vesicles towards the plasma membrane exocytotic site.

Five speakers in one session highlighted the role of different chromogranin-derived peptides, such as vasostatins, pancreastatin, catestatin, serpinins, and other fragments in health and disease. Granins, in addition to having intracellular functions, e.g., in secretory granule biogenesis, have extracellular functions after release in the secretory cell microenvironment and in the circulation. Indeed, a growing body of evidence suggests that certain granins, such as $\mathrm{CgA}$, $\mathrm{CgB}$, and $\mathrm{SgII}$ are proteolytically processed to generate peptides that have diverse extracellular biological activities. In particular, Sushil K. Mahata (USA) discussed the emerging role of chromogranin A-derived peptides pancreastatin and catestatin in the metabolic syndrome. Laurent Yon
(France) showed data suggesting that chromograninderived peptides can be exploited as markers for the diagnosis and prognosis of pheochromocytoma. In addition to Chromogranin A-derived peptides, Peng Loh (USA) showed that a splice variant of carboxypeptidase $\mathrm{E}, \mathrm{CPE}-\Delta \mathrm{N}$ is a powerful prognostic biomarker for predicting future metastasis in pheochromocytoma patients.

Angelo Corti (Italy) discussed the role of chromogranin A and its N-terminal fragment (vasostatin-1) in the regulation of endothelial barrier function, tumor vascular physiology and response to therapy. Peng Loh showed data supporting a role of serpinin, a peptide derived from the $\mathrm{C}$-terminal region of chromogranin A in secretory granule biogenesis and neuroprotection. Bruno Tota (Italy) showed new data suggesting that vasostatin, catestatin, and serpinin can exert different and sometimes opposite effects on heart contractility, which may be important for the regulation of cardiovascular homeostasis in normal and pathological conditions. In addition, Daniel O'Connor (USA) described many naturally occurring genetic variations in $\mathrm{CgA}, \mathrm{CgB}$, SgII, NPY, and NPY receptor genes in humans that disrupt the interaction of transacting factors to regulatory motifs in the genes, resulting in altered gene expression. These variants in those people render them to cardio-renal disease risk. The studies of Mahata, O'Connor, Corti, Loh, and Tota reported at the conference are summarized in a review below.

Thus, it is evident from all the presentations cited above that significant advancements have been made in the understanding of the mechanisms involved in regulating dense core granule biogenesis, transport, docking and exocytosis, and the pivitol role the cytoskeletal network plays in the latter processes. It has also become increasingly clear that the peptides derived from $\mathrm{CgA}$ play very important roles as extracellular signaling molecules to mediate different physiological functions, from neuroprotection in the brain to regulating glucose homeostasis, cardiac contractility, the autonomic system and tumor angiogenesis. 